\title{
DIVERSIDAD, DIFERENCIA Y ACCESIBILIDAD: ENFOQUES EDUCATIVOS EN EL DISCURSO DE LA DIVERSIDAD
}

\author{
Orlando Terré Camacho ${ }^{1}$ \\ Giselle Miranda Cervantes ${ }^{2}$
}

Hay momentos en la vida en los cuales la cuestión es saberse, se puede actuar diferente de lo que se piensa, y percibir diferente de lo que se ve, pero se hace imprescindible para seguir: mirar y reflexionar.

\section{Resumen}

Este artículo plantea la necesidad ineludible de compartir la construcción de un nuevo escenario escolar, más diverso y más plural, que permita adoptar propuestas y soluciones de acuerdo con los diferentes contextos sociales y culturales propios para establecer líneas prioritarias basadas en la equiparación de oportunidades y la igualdad de derechos.

Plantea la necesidad de reformular, cuántas veces sea necesario, el concepto de diversidad, para asumir y valorar las múltiples necesidades del individuo y hacer de las mismas la base fundamental de la planificación de la sociedad, en la cual la accesibilidad sea el proceso en el que se potencian todas las áreas del individuo, mediante el cambio de actitudes y las adaptaciones necesarias basadas, fundamentalmente, en el logro de la máxima funcionalidad y autonomía.

Palabras claves: diversidad, educación, accesibilidad.

1 Defectólogo con distinción Máster in Art of Education de la Universidad de Moscú-Rusia. Actualmente es Presidente de la Asociación de Educación Especial, Presidente Ejecutivo de la Órganización Mundial de Educación, Estimulación y Desarrollo Infantil (OMEDI) y creador del Método Point.

2 Licenciada en Psicología y egresada de la Maestría en Psicología de la Universidad de Costa Rica. Actualmente es Directora de la División de Educación Básica, Centro de Investigación y Docencia en Educación (CIDE), Universidad Nacional, donde se ha desempeñado como docente e investigadora desde 1990. 


\section{Abstract}

This article outlines the unavoidable necessity to share the construction of a new school: more diverse, more plural that allows to adopt proposals and solutions according to the different own social and cultural contexts to establish high-priority lines based on the comparison of opportunities and the equality of rights.

It outlines the necessity of reform, how many times it is necessary, the concept of diversity in order to assume and to value the individual's multiple necessities and to make of the same ones the fundamental base of the planning of the society, in which the accessibility is the process in the one that you powered the individual's areas by means of the change of attitudes and the necessary adaptations based fundamentally on the achievement of the maximum functionality and autonomy.

Keywords: diversity, education, accessibility.

\section{L}

as últimas tres décadas son testimonio de cambios y progresos ocurridos en el mundo en relación con la Educación Especial. En ella, ha prevalecido, por una parte, la intención de búsquedas de métodos de enseñanza eficaces para la intervención de los niños y las niñas con trastornos y alteraciones en el desarrollo, la participación activa de la familia, la escuela y la comunidad en la formulación de una comunidad científica educativa y, por otra, la aplicación de las altas tecnologías como herramientas importantes para la detección, el diagnóstico y la intervención de los déficits en el desarrollo.

Estos progresos y cambios se han encaminado con el propósito de establecer un tipo de escuela capaz de adaptarse, acoger y cultivar las diferencias como un elemento de valor positivo y la apertura de un espacio pluralista y multicultural donde se mezclen los colores, los géneros, las capacidades. Una escuela donde la heterogeneidad es asumida como una característica de todo grupo humano y, por lo tanto, su objetivo fundamental es hacer explícito lo implícito. Tal hecho permite a las personas el acceso a los servicios básicos elementales y a la construcción de una escuela, de una educación, donde cada quien, sin exclusión, encuentre una respuesta educativa acorde a sus necesidades y características peculiares.

Se constituye también en una escuela para todos, abierta a la diversidad, pero sin perder la perspectiva que la diversidad es siempre mucho más diversa de lo que podemos imaginar.

Hemos asistido en los últimos tiempos, sin lugar a dudas, a situaciones esperanzadoras, pero también de preocupación, en torno a cuestiones como, 
¿cuál debe ser la nueva escuela?, ¿qué tipo de enseñanza puede responder a los soportes del nuevo siglo? y ¿qué sistemas educativos y nomenclaturas deben perfilar la atención a los niños y las niñas con desventajas educativas y sociales?

El tema de atención a la diversidad presupone una propuesta que va de lo imposible a lo posible: el desespero de educar en la diversidad no es un tema nuevo; la escuela que hemos conocido y en la que nos han educado ha sido una escuela donde la práctica inclusiva ha aceptado algunas de las diferencias individuales, ha valorado a cada niño y niña, y le ha permitido la convivencia y la cooperación no totalitaria. Es desde este postulado que la actualidad del problema radica en convertir la escuela actual en un espacio donde la diversidad sea una regla y no una excepción. Una escuela en donde la diversidad es entendida como una oportunidad para aprender de la variedad humana $y$, por lo tanto, de la condición misma del ser humano en toda su dimensión.

Nuestros países, nuestras naciones, sufren las consecuencias de una fuerte crisis económica producida por el hegemonismo de políticas neoliberales ahora globalizadas. No es para menos el incremento de la pobreza y de las desigualdades del mundo, sobre todo en los últimos veinte años (los de hegemonía de la revolución conservadora) cuando, es tan espectacular, que amenaza con deslegitimar todo el proceso de globalización y hasta el paradigma de las nuevas economías se ve involucrado de manera especial.

Los sistemas educativos de nuestros países, unidos a las capas más pobres de las sociedades en vías de desarrollo, cargan con los mayores sufrimientos, unas veces porque repercuten en su vida personal y otras, porque su labor profesional se ve lastimada por la ausencia de nuestros niños y niñas en la escuela, dadas las precarias situaciones que distan de las posibilidades y el derecho a la educación y a la vida. Sin embargo, ninguno de los sacrificios hechos hasta ahora, alcanzarán sus frutos, si no logramos adquirir mayor conciencia de los desafios que vive el mundo.

La escuela contemporánea se ve preocupada con la efervescencia que la envuelve. Verificamos que la rapidez y la velocidad del mundo se nos presentan en fragmentos, algo parecido a un rompecabezas, que por la pluralidad de sus piezas parece dificil de armar. No obstante, se hace imprescindible compartir la construcción de un nuevo escenario escolar, diverso y plural, que permita adoptar propuestas y soluciones según los diferentes contextos sociales y culturales propios para establecer líneas prioritarias basadas en la equiparación de oportunidades de las personas portadoras de necesidades especiales y la aportación de igualdad de derechos. 


\section{El significado de la igualdad}

Los términos diversidad e igualdad son principios y valores básicos, reconocidos explícitamente desde la Modernidad, como presupuestos para alcanzar una convivencia ordenada y articulada que gire en torno al principio de Justicia, entendida esta como equidad y que, como tal, demanda la coexistencia y el respeto a ambos principios: el de la igualdad y el de la diferencia.

La Asamblea General de las Naciones Unidas, a través de la Declaración Universal de Derechos Humanos, hacía saber el 10 de diciembre de 1948 en el artículo I que: "Todos los seres humanos nacen libres e iguales en dignidad y derecho...". Y en su artículo 7 especificaba: “... todos son iguales ante la ley y tienen, sin distinción, derecho e igual protección...”. Esto patentiza una posición clara contra la discriminación y en pro de la diversidad y la integración de todas las diferencias y si bien la legislación no es el único medio de progreso social, representa uno de los más poderosos vehículos de cambio, adelanto y desarrollo de la sociedad.

En este contexto, afirman Devalle y Vega (1999):

Si la escuela no está abierta a la diversidad, si no hay igualdad de oportunidades, basada simplemente en la dignidad de la persona y en el derecho humano inalienable a la educación, una educación en y para la diversidad corre el riesgo de serlo solo para los privilegiados que acceden a la escuela (p. 11).

Si es preciso, y para hacer frente a tal despropósito, ha de reformarse, cuántas veces sea necesario, el concepto de diversidad, apoyándonos en el principio de igualdad que comporta necesariamente el reto de asumir y valorar la diversidad en la Educación, en la Cultura y la Sociedad, al reconocer la importancia de las diversas necesidades de cada persona y hacer de estas la base fundamental de la planificación de la sociedad.

En este sentido, lo más importante, a nuestro juicio, no radica en definir una opción terminológica que describa el proceso de atención a las personas con necesidades educativas especiales, sino en describir las posibilidades que se puedan brindar teniendo en cuenta las condiciones histórico-concretas particulares, otorgando el derecho que tienen todos los seres humanos, en consecuencia, a ser considerados en cada caso siempre como fin y nunca instrumentalizados como medios. 


\section{Mas allá de la integración}

Una revisión completa de los rasgos conceptuales e ideológicos del fenómeno de la integración educativa refiere a algo más complejo de lo que puede suponerse, pues pone en evidencia los sistemas educativos tradicionales y su aspiración a convertirse en un modelo totalmente nuevo, renovado, flexible, abierto, que responda a las necesidades educativas de todos los educandos. La integración educativa debe partir precisamente de la posibilidad que le brinda la sociedad a cada individuo de integrarse en ella con iguales derechos, aunque con posibilidades diferentes.

La comprensión de la diferencia se establecerá en la manera que asumamos que cada uno de nosotros es una persona única, cada una es diferente por la interacción entre lo que es (intelectual, motivación, intereses, experiencias acumuladas y conocimientos) y por su ubicación en el contexto (social, ambiental y cultural).

En el contexto de la integración, resulta difícil dejar de mencionar el tema de la diversidad y asumirla supone reconocer el derecho a la diferencia como medio para el enriquecimiento educativo y social. Supone también que los procesos educativos formales deben tender a la igualdad y a la justicia social, respetando, pero sobre todo, potenciando las diferencias.

El reto de la atención a la diversidad en la escuela es un tema que ha estado presente desde los inicios del desarrollo de la ciencia pedagógica, formulada en el clásico principio pedagógico de la atención a las diferencias, que encuentra su marco histórico en la Grecia antigua y que hoy el planteamiento es mucho más complejo.

Evidentemente, las políticas y las acciones que se apoyan en el discurso de la diversidad deben ir acompañadas de una realidad de igualdades (Sáez, 1997). De lo contrario, se corre el riesgo de asumir la igualdad y la diversidad aceptando una escuela en la que prevalece la selección y en la que se legitiman aún más las exclusiones, materializadas en la carencia de condiciones básicas y de códigos para la realización de derechos junto a un mal sentido de cooperación y reciprocidad como la base del bien común (Macal, en Cordero, 2004, p. 25).

La atención a la diversidad se constituye en un poderoso referente para reactivar las tensiones, los dilemas y los retos, que están afectando a muchos países occidentales y sociedades que se declaran, cada vez con más énfasis, a favor de la justicia; pero las gestoras del mercado, impulsoras de la rentabilidad y de la eficacia, promueven multitud de desigualdades, una distribución 
injusta de los recursos, altas cuotas de pobreza y nuevas formas de discriminación; situación ante la cual, la atención a la diversidad puede ser un tópico, un hermoso eslogan o una preocupación que promueva la dimensión comprometida del ser humano.

La pobreza y las desigualdades no son un fenómeno natural, sino fruto de políticas económicas equivocadas o defensoras de los intereses de los más poderosos y presupone identificar si la lucha por la diversidad y la nulidad de la pobreza es auténtica o sólo es un instrumento más de propaganda. Por esta razón, se debe procurar que los procesos educativos formales sean deconstruidos, analizados y reconstruidos con miras a la consecución de la igualdad de oportunidades para todos los seres humanos (Sales y García, 1997).

Otro término que ocupa vigencia es el de la accesibilidad a los servicios esenciales de educación y trabajo, como una forma de integrarse a la comunidad y no sentirse rechazado por ella. Por esta razón, se potencian todas las áreas del individuo mediante el cambio de actitudes y de las adaptaciones necesarias que tienen como base fundamental el logro de una máxima funcionalidad y autonomía. Para las personas con algún tipo de discapacidad, la falta de acceso a los servicios básicos sigue siendo una fuente de discriminación y pérdida de oportunidades.

La posibilidad de acceder a los servicios sociales es un requisito previo para las personas con discapacidad, que les permitirá no sólo gozar de igualdad de oportunidades, sino que se constituye en la llave para ejercer plenamente sus derechos civiles, sociales, políticos y culturales, otorgando el derecho de igualdad de todos los seres humanos. No debemos olvidar que el $80 \%$ de las personas con algún tipo de discapacidad en el mundo vive en países en vías de desarrollo.

\section{Enfoques educativos y líneas de investigación en torno a la diversidad}

La educación presupone el planteamiento de modificaciones significativas de la institución educativa y de lo que en ella ocurre, es decir, es necesario introducir modelos de atención a la diversidad en las estructuras de la organización y revisar toda la organización en las instituciones educativas.

De la idea del cambio y el mejoramiento de los sistemas educativos se postulan diferentes criterios, que basan su objetivo en el nacimiento de distintas iniciativas en relación con las reformas educativas, que encuentran sus primeros intentos en las décadas de los sesenta y los setenta y que, a juicio de los estudiosos del tema, ponen de manifiesto dos hechos (Sáez, 1998): 
- Los evidentes fracasos de las reformas liberales para llevar a cabo sus programas y cumplir sus objetivos adecuadamente.

- La aparente incapacidad de los reformadores de la escuela liberal para explicar adecuadamente sus fracasos reformistas, sin imaginación para utilizar en sus evaluaciones otro conocimiento que no sea el técnico.

\section{Estudios de eficacia}

Las primeras investigaciones realizadas en torno a los principios en los que se apoya la ideología de la normalización se han dado a conocer en la literatura especializada con la denominación de "estudios de eficacia". La mayor parte de estos estudios fueron llevados a cabo en Estados Unidos y en el período previo a la promulgación de su Ley de Integración en el año 1975.

Los estudios de eficacia se definen por un intento de evaluar la relativa eficacia de un determinado tipo de ubicación educativa -aula regular/aula especial- sobre el rendimiento académico y los logros socioemocionales de los y las estudiantes con necesidades especiales. Cada uno de ellos, es igualado con base en una serie de características previsiblemente homogéneas (C.I., edad cronológica, sexo), para después ser situados en los dos tipos de ubicación que pretenden ser contrastados. La población objeto de estudio se encuentra circunscrita a aquellos estudiantes incluidos en la categoría de deficiencia mental ligera y media, con trastornos de aprendizaje y con trastornos emocionales.

En conjunto, estas investigaciones van a tratar de dar una respuesta a la necesidad de probar, dado el clima social imperante, los beneficios de la integración para promover el progreso académico y social de los y las estudiantes en contextos integrados. Beneficios, o no, que van a ser identificados a partir de diseños de investigación, más preocupados por los resultados que por los procesos y condiciones de aprendizaje que se desencadenan en los marcos educativos que se someten a contraste.

Diseños metodológicos que, en su momento, se vieron sometidos a fuertes críticas debidas, fundamentalmente, a la confusión y conflictividad entre los datos obtenidos acerca de un mismo tipo de análisis (rendimiento académico y autoconcepto, entre otros).

Tal y como señala McHillan (1971), la escasa información que se ofrece desde estos estudios respecto a la selección de los y las estudiantes identificados como "especiales" lleva a considerar la existencia de una cierta propensión selectiva en el muestreo. Es decir, que tienden a ubicar en las aulas ordinarias 
a aquellos alumnos que podrían estar próximos a la normalidad. Sesgo que cabría considerarse como un factor explicativo de la tendencia aportada por estos estudios relativa a la superioridad del aula regular frente a la especial.

Indiscutiblemente, múltiples son las investigaciones que nos acercan al intento de debatir una temática, importante y necesaria, como la que nos ocupa; y dejar de mencionar un conjunto de estas investigaciones no significa, en ningún caso restarle su valor, pero las mencionadas y las no mencionadas dejan claro que:

- Atender la diversidad es favorecer la convivencia de realidades plurales, de necesidades diferentes, que enriquezcan la dinámica del aula y la institución.

- El simple cambio de lugar de los y las estudiantes de un aula especial a un aula regular, no produce por sí mismo, un aumento académico y social y, mucho menos, emocional.

- Proporcionar a la o el estudiante con necesidades educativas especiales programas, materiales, sistemas de apoyo adaptados a sus necesidades no presupone el reto que nos proponemos.

Atender y aceptar la diversidad tiene complejas implicaciones que nos inducen a pensar que no podremos educar en la diversidad sin cambiar la educación y las sociedades que nos acompañan; para ello, debemos tener en cuenta que:

- La formación del profesorado debe traspasar los límites legales, convirtiéndose no sólo en un dador de conocimiento, sino en un modelador de la zona de aprendizaje de cada educando.

- Se deberá facilitar y promover la flexibilidad curricular.

- Establecer espacios de comunicación entre centros educativos, profesorado, comunidad y alumnado, con espacios adecuados de convivencias.

- Promoción y concienciación de las comunidades, con el fin de desempeñar una labor divulgativa en relación con el reto de participación comunitaria en unidad por la diversidad.

- $\quad$ Promover grupos de investigación estables en departamentos, centros educativos y universidades.

- Diseñar proyectos que apoyen al desarrollo profesional, potenciando el trabajo colaborativo como apuesta ideológica para la solución de problemas. 
- Conseguir que, a través de la educación institucionalizada, seamos capaces de ayudar al niño o a la niña a crecer y a desarrollarse como persona, facilitándole la adquisición de habilidades básicas (cognoscitivas, autoconocimiento, autonomía personal y socialización).

- Facilitar que en las instituciones educativas tengan cabida y reconocimiento todas las diferentes capacidades, los ritmos de trabajo, las expectativas, las motivaciones, las etnias y los valores culturales de todos los actores de la escena educativa.

\section{Conclusiones}

Se hace evidente que la aproximación a los temas de la diversidad, la diferencia y la accesibilidad y EDUCAR EN LA DIVERSIDAD presuponen la aplicación de un nuevo paradigma, de postulados científicos vigentes y de visión en relación con los sistemas educativos y las sociedades actuales.

Existen retos inmediatos; uno de ellos es adaptar la enseñanza a la diversidad de niños y niñas que conviven en sociedades pluralistas y adecuar las políticas y acciones que se apoyan en el discurso de la cultura por la diversidad que, necesariamente, deberán ir acompañadas de una realidad de igualdades y del verdadero sustento social que esta exige y de las que le son imprescindibles.

El paso debe ser reflexionar y superar los antiguos discursos, plagados de criterios y conceptos de tiempos viejos. Podemos adecuarnos a los nuevos tiempos si somos capaces de dar participación activa y dinámica a la comunidad, propiciando un marco de acción plena y posibilitando un ambiente de comunicación abierta y flexible entre todos los entes participativos. En este sentido, la respuesta a la diversidad no ha de ser una decisión puntual, ni asumida por individualidades, ni tampoco debe ser solucionada únicamente con ayudas externas; el planteamiento ha de ser institucional desde el plano escolar hasta el social.

En cualquier caso, existe la necesidad y la posibilidad de lograr una escuela, una enseñanza y una sociedad, donde las diferencias no sean un lastre formativo sino de crecimiento individual y social. Nos urge una mejor idea pensada en el enriquecimiento formativo y de crecimiento individual y social de todos los seres humanos, de cara a asumir reflexivamente las vivencias, los derechos y las obligaciones como miembros activos de una escuela universal y como ciudadanos de una sociedad democrática.

De hecho, estas y algunas otras variables nos dejan la oportunidad de reflexionar en torno a que, educar en la diversidad, es hoy por hoy una necesidad inconclusa. 


\section{Referencias}

Amaiz, P. y Illán, N. (1988). "La integración en el marco de la Reforma de la enseñanza". Jornadas sobre la Reforma del sistema educativo, 129-143.

Amaiz, P. y Lozano, J. (1996). Proyecto curricular para la diversidad. Madrid: CCS.

Brennan, W. K. (1988). El currículo para niños con necesidades especiales. Madrid: SIGLO XXI.

Bruner, J. S. (1988). Desarrollo cognitivo y educación. Madrid: MORATA.

Camps, V. (1994). Los valores de la educación. Madrid: ALAUDA.

Cascón, P. (1993). Igualdad para vivir, diversidad para convivir: educar para no discriminar. L'Hospitalet de Lobregat: Ajuntament de L'Hospitalet de Llobregat.

Cool, C. y otros. (1990). Aprendizaje escolar y construcción del conocimiento. Buenos Aires: PAIDÓS.

Cool, C. y otros. (1992). Los contenidos de la Reforma. Madrid: SANTILLANA.

Cordero, H. (2004). "Educación inclusiva". Revista EDUCARE, 6(1), 23.

Delval, J. (1994). El desarrollo humano. Madrid: SIGLO XXI.

Devalle, A. y Vega, V. (1999). Una escuela en y para la diversidad: el entramado de la diversidad. Argentina: AIQUE, Grupo Editor, S. A.

Dewey, J. (1916). Democracy and education. New York: FREE PRESS.

Escudero, J. M. (1995). "Los temas transversales y las reformas educativas: posibilidades y limitaciones". Ponencia presentada al Curso de Verano sobre "La transversalidad en la Reforma Educativa". Universidad de Mar/ Universidad de Murcia. Agulas, Julio.

García, J. A. (1990). “Un objetivo pedagógico: Educar para la Paz". Revista de Pedagogía Social (5).

Illán, N. (1989). Los profesores y la integración escolar. Valencia: NAU LLIBRES.

M.E.C. (1990). Evaluación del Programa de Integración. Madrid: MEC.

M.E.C. (1990). Orientación educativa e intervención psicopedagógica. Madrid: MEC.

Sáez, J. (1989). La construcción de la educación. Valencia: NAU LLIBRES.

Sáez, J. (1993). "Las condiciones cognitivas de la democracia". En Ortega, P. y Sáez, J. Educación y democracia. Murcia: CAJAMURCIA.

Sales, A. y García, R. (1997). El fenómeno multicultural: origen y fundamentos ideológicos. Bilbao, España: DESCLÉE DE BROUWER, S. A.

Terré, O. (1998). Actualidades en educación especial. Lima-Perú: EL LIBRO AMIGO.

Terré, O. (2000). Variables entorno a la educación y la diversidad. Discurso VI Encuentro Mundial de Educación Especial, Saltillo-Coahuila-MÉXICO.

Vygotsky, L. (1979). El desarrollo de los procesos psicológicos superiores. Barcelona: CRÍTICA. 